\title{
Asthma drug adherence in a long term clinical trial
}

Gunnar Jónasson, Kai-Håkon Carlsen, Petter Mowinckel

\begin{abstract}
Aim-To measure drug adherence in children with mild asthma receiving long term prophylactic treatment.

Methods-Double blind randomised placebo controlled trial. Patients received inhaled budesonide $100 \mu \mathrm{g}$ or $200 \mu \mathrm{g}$ daily, or placebo for 27 months. All participants were asked to inhale medication or placebo from two different Turbuhalers (morning and evening) during the study. A total of 122 children ( 80 boys, 42 girls) aged 7-16 years with mild asthma (mean FEV $_{1}$ $103.7 \%$ of predicted) were included in the trial. Drug adherence was assessed by counting the number of remaining doses in the inhaler when study medication was returned at six month intervals.

Results-A statistically significant and continuing decrease in measured drug adherence was found from three to nine months and then to 27 months, reaching mean values of $40.6 \%$ and $46.9 \%$ for inhaled morning and evening medication respectively. Drug adherence declined more rapidly in the placebo group (compared to active treatment); this difference became significant after two years of treatment. Children aged 9 years or less had better drug adherence during the entire study period, but the difference was only significant for the first three months of the study. Measured drug adherence was significantly higher for evening medication compared to morning medication for all study intervals after nine months. Conclusion-Measured drug adherence diminishes significantly when treating children with mild asthma in a long term trial. This emphasises the importance of monitoring compliance in clinical trials. (Arch Dis Child 2000;83:330-333)
\end{abstract}

Keywords: drug adherence; patient compliance; inhaled corticosteroids; childhood asthma

Voksentoppen Centre of Allergy, Asthma and Chronic Lung Diseases in Children, Oslo,

Norway

K-H Carlsen

Health Services Department, Central Hospital of Akershus, Norway

P Mowinckel

Correspondence to: Dr Jónasson

email: gionasso@online.no

Accepted 8 June 2000 authors' opinion this will inevitably lead increased attention on drug adherence in this group of patients.
Drug adherence can be determined directly by repeated measurement of serum concentrations of the drug (or a metabolite) in question, when methods are available (serum theophylline measurements). Treatment adherence can also be indirectly measured objectively by assessing the use or intake of prescribed medication. This has been assessed electronically in a clinical trial concerning the use of inhaled steroids for 13 weeks in childhood asthma and was reported to be $58 \% .^{3}$ Few studies have however reported objectively measured drug adherence in a long term trial involving children with asthma. Prescribing prophylactic long term therapy in children with mild asthma in order to treat inflammation in the airways and obtaining symptom control may possibly result in a decline in treatment adherence.

\section{Patients and methods}

STUDY DESIGN

The study was a double blind, placebo controlled single centre extension trial. The study was a direct continuation of a previous 12 week trial $(n=163)$, which has been reported and described in detail elsewhere. ${ }^{4}$ At baseline patients were randomised into four different treatment groups, and each patient received two Turbuhalers, one for morning medication or placebo and the other for evening medication or placebo. Group I patients were given budesonide $100 \mu \mathrm{g}$ once daily in the morning and placebo in the evening. Group II received budesonide $200 \mu \mathrm{g}$ once daily in the morning and placebo in the evening. Group III received budesonide $100 \mu \mathrm{g}$ twice daily. Group IV received placebo twice daily. Clinical assessments were performed at three month intervals during the first year and at six month intervals during the second year.

PATIENTS

A total of 122 children with mild asthma were included in the present study, 80 boys and 42 girls, aged 7-16 years. All were selected from the outpatient clinic at the Section of Allergy and Pulmonology, Department of Paediatrics, Ullevål University Hospital in Oslo. All subjects participated in a two year extension study on the effect of low dose inhaled budesonide on mild asthma, mild being defined as low grade symptoms that did not interfere with sleep and lifestyle, or episodes of cough and wheeze occurring less than once per month. ${ }^{5}$ Table 1 gives demographic details of participants.

\section{METHODS}

Patient drug adherence was assessed by counting the remaining doses in the inhaler device which initially contained 200 doses. This was 
Table 1 Patient characteristics at baseline

\begin{tabular}{|c|c|c|c|c|}
\hline Characteristics & $\begin{array}{l}\text { BUD } 100 \mu g \\
\text { twice daily }\end{array}$ & $\begin{array}{l}\text { BUD } 200 \mu g \\
\text { once daily }\end{array}$ & $\begin{array}{l}\text { BUD } 100 \mu g \\
\text { once daily }\end{array}$ & Placebo \\
\hline Number of patients & 28 & 32 & 28 & 34 \\
\hline Boys/girls & $18 / 10$ & $17 / 15$ & $23 / 5$ & $22 / 12$ \\
\hline Age $(y)$ & 10.2 & 10.0 & 9.5 & 9.4 \\
\hline Height $(\mathrm{cm})$ & 145.6 & 145.6 & 139.3 & 138.7 \\
\hline Number of atopics & 20 & 21 & 20 & 26 \\
\hline $\mathrm{FEV}_{1}(1)$ & $2.27(0.57)$ & $2.23(0.66)$ & $2.30(0.85)$ & $2.04(0.67)$ \\
\hline $\mathrm{FEV}_{1} \%$ of pred & $106.0(14.1)$ & $102.1(11.4)$ & $102.2(12.8)$ & $104.6(12.9)$ \\
\hline Reversibility in $\mathrm{FEV}_{1}$ (\%) & $4.3(3.8)$ & $3.6(3.4)$ & $2.4(2.8)$ & $3.8(3.1)$ \\
\hline PEF morning $(1 / \mathrm{min})$ & $263.8(62.2)$ & $260.4(84.2)$ & $259.9(85.4)$ & $229.5(75.9)$ \\
\hline Methacholine $\mathrm{PD}_{20}(\mu \mathrm{mol})$ & 3.75 & 4.33 & 5.83 & 4.04 \\
\hline $\begin{array}{l}\text { Maximum fall in FEV } \\
(\%) \text { after exercise test }\end{array}$ & $10.6(8.1)$ & $13.7(12.4)$ & $14.7(17.5)$ & $10.1(9.6)$ \\
\hline
\end{tabular}

Results are given as mean (SD).

$\mathrm{BUD}$, budesonide; $\mathrm{FEV}_{1}$, forced expiratory volume in 1 second; $\mathrm{PEF}$, peak expiratory flow; $\mathrm{PD}_{20}$ provocative dose causing a $20 \%$ fall in $\mathrm{FEV}_{1}$.

done at six monthly intervals or when the inhalers were returned. This method has been described in detail elsewhere. ${ }^{4}$ If inhalers were lost during the study period, patients were provided with new inhalers for the rest of that six month period, but these patients were excluded from the measurement period.

Drug adherence was calculated as:

$\%$ drug adherence $=$

$\frac{200-\text { number of remaining doses } \times 100}{\text { number of prescribed doses }}$

Patients were not informed of compliance measurements and the results of these were unknown to the study investigator until the study was completed. The study was approved by the regional medical ethics committee.

STATISTICAL EVALUATION

Means were used for the index of location and confidence interval as the index of dispersion. Unpaired $t$ tests were used to compare drug adherence between different groups, and paired $t$ tests were used to compare the difference between morning and evening compliance. Probability values of 0.05 and less were considered to be significant.

\section{Results}

PATIENTS

Thirty three patients withdrew during the study period: 19 because of disease deterioration, 14 because of non-compliance, and one because of change of residence. After three months of treatment all inhalers $(n=244)$ were recovered for measurement from included study subjects. From 9 to 21 months, $94-98 \%$ of inhalers of included patients were recovered for measurements, whereas $69 \%$ of inhalers (123 of 178) were included in the measurement at the last visit of the study.

Table 2 Comparing measured compliance when treating mild childhood asthma with twice daily inhaled budesonide or placebo for 27 months

\begin{tabular}{llllll}
\hline & Budesonide & $n$ & Placebo & $n$ & p value \\
\hline 3 months & $76.9(72.3-81.4)$ & 87 & $78.1(69.1-87.0)$ & 34 & 0.79 \\
9 months & $54.2(48.0-60.4)$ & 83 & $57.1(47.8-66.4)$ & 32 & 0.61 \\
15 months & $53.2(46.7-59.7)$ & 77 & $44.7(33.4-55.9)$ & 27 & 0.18 \\
21 months & $50.5(43.4-57.5)$ & 71 & $36.2(21.8-50.5)$ & 23 & 0.054 \\
27 months & $48.8(39.8-57.8)$ & 44 & $32.2(15.8-46.6)$ & 19 & 0.039 \\
\hline
\end{tabular}

Mean values are given as percentages $(95 \% \mathrm{CI})$.
DRUG ADHERENCE ASSESSMENT

Mean measured adherence for morning medication dropped significantly $(p<0.05)$ for twice daily medication: from $76.7 \%$ after three months of treatment to $51.7 \%$ six months later for morning medication, and respectively from $77.7 \%$ to $58.8 \%$ for evening medication in the same period. As table 2 shows, mean adherence for twice daily medication continued to diminish throughout the study period. Patients receiving placebo had higher (but not significantly) drug adherence during the first nine months of the study, and thereafter consistently lower adherence when compared to active treatment to the end of the period. This difference was statistically significant during the last six months of the study as shown in table 2, which presents the mean adherence values per patient for joined morning and evening medication, with the lowest adherence value for the placebo group at the end of the study $(32.1 \%)$ compared to $48.7 \%$ for budesonide treatment. Figure 1 shows similar trends for the comparison of adherence to morning medication only.

Adherence in two different age groups (7-9 years versus $10-16$ years at baseline) was on all occasions higher in the younger age group. This difference was however only significant for the first three months of treatment as presented in table 3 for evening medication and in fig 2 for morning medication.

Measured adherence was always higher for evening medication; this difference was statistically significant on all visits after three months of treatment as shown in table 4 .

\section{Discussion}

The present study shows that measured drug adherence in a clinical trial involving children with mild asthma, diminishes significantly with time in a long term study. After nine months of treatment, drug adherence was found to be constantly better in the group of patients that inhaled a low dose of budesonide compared to placebo with significant difference the last six months of the study. Adherence was also found to be significantly better for evening medication at nine months and towards the end of the study. Younger children (7-9 years) had a significantly higher adherence to the inhalation therapy during the first three months of the study, possibly as a result of parental motivation, but this difference seems to be reduced as the adherence decreases.

This study emphasises the importance of monitoring compliance in a clinical trial. This is important with regard to the assessment of a possible dose-response effect of a given treatment; it can also influence the general conclusion to be drawn from a trial, as has been pointed out by others. ${ }^{6}$ To the authors' knowledge, a long term clinical trial, where drug adherence to asthma medication has been objectively measured for more than two years, has not been reported previously.

As with all other indirect measurements of compliance-counting unused blisters or pills, or using an electronic inhaler timer device (Nebulizer Chronolog Forefront Technologies Inc., Lakewood, Colorado, USA) - the method 


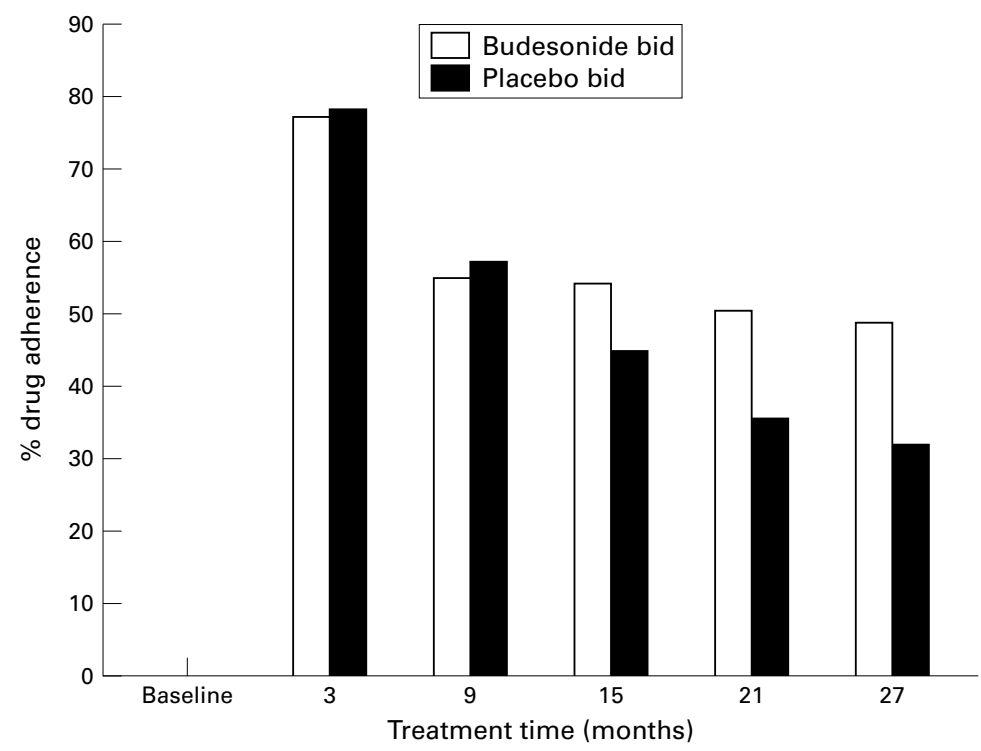

Figure 1 Measured drug adherence for use of Turbuhaler in the morning, treating mild childhood asthma with inhaled budesonide or placebo for 27 months.

Table 3 Comparing measured drug adherence for evening medication between two age groups of children with mild asthma treated with inhaled budesonide or placebo for 27 months

\begin{tabular}{llllll}
\hline Months & Age 7-9 & $n$ & Age 10-16 & $n$ & p value \\
\hline 3 & $81.8(78.2-85.4)$ & 61 & $71.6(63.2-79.8)$ & 61 & 0.001 \\
9 & $62.2(55.3-69.1)$ & 59 & $55.2(47.2-63.1)$ & 55 & 0.18 \\
15 & $57.0(49.6-64.3)$ & 55 & $53.2(43.7-62.8)$ & 46 & 0.53 \\
21 & $51.9(42.5-61.3)$ & 48 & $48.6(38.1-59.0)$ & 45 & 0.63 \\
27 & $53.1(42.0-64.1)$ & 32 & $40.1(28.1-52.1)$ & 29 & 0.30 \\
\hline
\end{tabular}

Mean values are given as percentages $(95 \% \mathrm{CI})$.

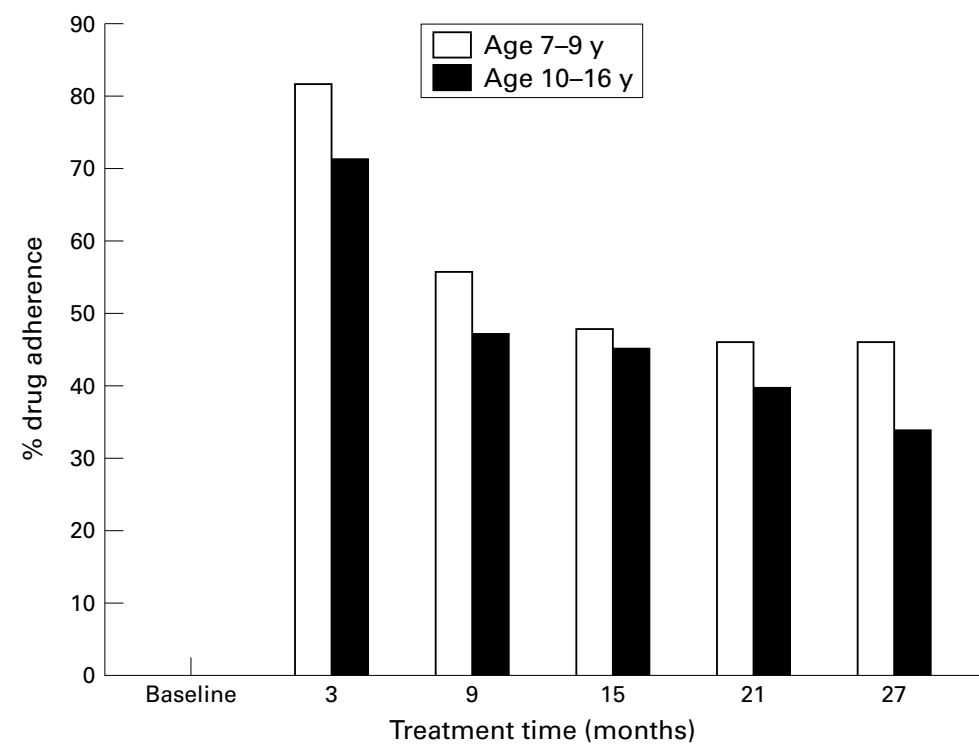

Figure 2 Comparing measured drug adherence in the morning between two age groups of children with mild asthma treated with inhaled budesonide or placebo for 27 months.

Table 4 Comparing measured drug adherence between morning and evening doses, when treating mild childhood asthma with inhaled budesonide or placebo for 27 months

\begin{tabular}{llllrl}
\hline Months & Morning & $n$ & Evening & $n$ & p value \\
\hline 3 & $76.7(72.2-81.2)$ & 122 & $77.6(73.8-81.5)$ & 122 & NS \\
9 & $51.7(46.4-57.0)$ & 115 & $58.8(53.6-64.0)$ & 114 & $<0.001$ \\
15 & $47.7(41.9-53.5)$ & 103 & $55.3(49.4-61.1)$ & 101 & $<0.001$ \\
21 & $43.6(37.2-50.0)$ & 93 & $50.3(43.4-57.2)$ & 93 & $<0.001$ \\
27 & $40.6(32.5-48.7)$ & 62 & $46.9(38.8-55.0)$ & 61 & 0.02 \\
\hline
\end{tabular}

Mean values are given as percentages $(95 \% \mathrm{CI})$. NS, non-significant. used in the present study will only reveal the "theoretical best" compliance, as it is not possible to rule out that the patient might have clicked the inhaler without inhaling ("test clicking"), as has been described by others, ${ }^{7}$ in which case the real compliance would be even lower than measured.

Some short term studies on drug adherence have reported overuse of medication, ${ }^{48}$ but it cannot be ruled out that this overuse is in some part at least caused by "test clicking" the inhaler. In the present study overuse of medication was not detected at all after the first three months, possibly suggesting that this effect wears off in a long term study as could be expected when adherence gradually diminishes with time, as in the present study.

Fourteen patients with "known" noncompliance were excluded from the study during the 27 month period. Some of these did not return their inhalers back to the clinic and consequently could not be included in the study for the last three or six months prior to their last visit in the study. The exclusion of these patients from the ongoing study can possibly have resulted in somewhat higher adherence results at the end of the study, than would be expected if they had not been excluded. It can therefore be concluded that drug adherence is likely to be underestimated in the present study.

Children participating in the present study all suffered from mild asthma. Our finding that drug adherence became significantly better in the treatment group when compared to placebo is an important observation, possibly suggesting that this low dose of an inhaled steroid was effective in controlling asthma symptoms, hence possibly resulting in a better adherence. Other studies have shown that failure to comply with asthma treatment results in increasing symptoms, ${ }^{3}$ but in the present study the main objective was to measure changing trend in concordance with prescribed inhalation therapy in mild asthma, rather than the efficacy of the treatment given.

It has been shown that participating in a clinical trial can increase the patient's motivation and compliance, ${ }^{9}$ and this conclusion is supported by the relatively high measured adherence at the beginning of the present study; nevertheless it shows clearly that patient adherence with the intake of inhaled steroids diminishes rather rapidly as time goes by, even in a clinical trial. We therefore conclude that it seems to be important to monitor patient compliance in order to understand how low it really can be, and find ways to improve it.

The authors thank A Sødal for her help in collecting the data. The study was supported by a grant from Astra Draco, Lund, Sweden, who delivered budesonide, terbutaline, and placebo Turbuhalers. 1 Warner JO, Naspitz CK. Third International Pediatric Con-
sensus Statement on the Management of Childhood Asthma. Pediatr Pulmonol 1998;25:1-17.

2 Vignola AM, Chanez P, Campell AM, Souques F, Lebel B, Enander I, Bousquet J. Airway inflammation in mild intermittent and in persistent asthma. Am F Respir Crit Care Med 1998;157:403-9. 
3 Milgrom H, Bender B, Ackerson L, Bowry P, Smith B, Rand C. Noncompliance and treatment failure in children with asthma. F Allergy Clin Immunol 1996;98: 1051-7.

4 Jonasson G, Carlsen KH, Sødal A, Jonasson C, Mowinckel P. Patient compliance in a clinical trial with twice daily inhaled budesonide or placebo in children with mild asthma. Eur Respir f 1999;14:150-4.

5 Warner JO, Neijens HJ, Landau LI. Asthma: a follow up statement from an international paediatric asthma consensus group. Arch Dis Child 1992;67:240-8.
6 Goldsmith $\mathrm{CH}$. The effect of compliance distribution on therapeutic trials. In: Haines RB, Taylor DW, Sackett DL, ds. Compliance in healthcare. Baltimore, MD: John

Hopkins University Press, 1979:297-308.
7 Rand CS, Wise RA, Nides M, et al. Metered-dose inhaler adherence in a clinical trial. Am Rev Respir Dis 1992;146: 1559-64.

8 Coutts JAP, Gibson NA, Paton JY. Measuring compliance with inhaled medication in asthma. Arch Dis Child 1991;67:332-3.

9 Reiser J, Warner JO. The value of participating in an asthma trial. Lancet 1985;26:206-7.

\section{0 people with Tourette syndrome}

The Tourette Syndrome International Database Consortium began in 1992 as a collaborative project between two centres in Canada and one in the USA, and now collects data from 65 centres in 22 countries on six continents. A recent report (Roger D Freeman and colleagues. Developmental Medicine and Child Neurology 2000;42:436-47) includes information on 3500 people with Tourette syndrome.

There was a preponderance of males at all centres, the overall male:female ratio being 4.3:1. Tics began in all cases before the age of 20 , in $41 \%$ before 6 years, and in $93 \%$ before 10 years. The diagnosis was made before the age of 10 in $56 \%$ and before the age of 15 in over $80 \%$. In only $12 \%$ of cases was Tourette syndrome the only diagnosis; other diagnoses included attention deficit hyperactivity disorder $(60 \%)$, obsessive compulsive disorder $(27 \%)$, obsessive compulsive behaviour (32\%), learning disorder (23\%), and conduct disorder/ oppositional defiant disorder (15\%). The number of comorbid disorders (in addition to Tourette syndrome) ranged from none to six (average two) and they strongly influenced the likelihood of behavioural problems - such as, anger control problems, coprolalia, self injury, and sleep difficulties, all of which were much more likely to occur with an increasing number of comorbid diagnoses. Thus, uncontrolled anger occurred in around 5\% of those with Tourette syndrome alone, about $40 \%$ of those with three additional diagnoses, and over $70 \%$ of those with six additional diagnoses.

Behavioural problems in people with Tourette syndrome should lead to renewed assessment for additional psychiatric diagnoses. Treatment of these comorbid conditions may be as important as treatment of the tics (or more important). The existence of this large database should facilitate future research.

ARCHIVIST 